\title{
Misdirection: model of information process for neuromagic
}

\author{
David Bestue \\ 1. Institut d'Investigacions Biomèdiques August Pi i Sunyer (IDIBAPS)
}

\begin{abstract}
Neuromagic is underpinned by the union of neuroscience and magic. However, the contribution between them is unbalanced. While neuroscience has used magic tricks to study different cognitive processes and develop sophisticated experiments, the returned contribution has not penetrated into magicians praxis. In neuroscience, a priori theories are supported by objective experimental data. However, this steps are switched in magic: subjective experimental evidence generates a posteriori theories. This scientifically invalid methodology leads, as a consequence, to several unvalidated theories about the same topic. To solve this problem, testing this theories with previously validated brain models is essential, as it will separate the ones that can be reproduced with realistic models from the ones that can not. Here, we use a popular brain model -the bump attractor- to describe the most studied magical principle: misdirection. Misdirection aims to avoid the detection of tricky maneuvers by affecting the attention and the memory of the spectators. Misdirection is been defined and classified in multiple ways for different magicians. For the first time, we will support with a model of neural activity, a recent misdirection classification (Bestue, 2019). The model reproduced the efficiency and provided a neural explanation for each of the three different subcategories of misdirection proposed by the classification: "direction", "division" and "diminishment". That way, we provided first neuroscientific evidence for a misdirection classification. We hope this work motivates both magicians and neuroscientists to provide detailed models to different magical principles, so they rely in objective brain functioning and not in subjective psychological theories.
\end{abstract}

\section{Introduction}

Neuromagic is a new field of study originated from the combination of magic and neuroscience (Kuhn 2019; Martinez-Conde \& Macknik 2008). The study of magic has helped neuroscientists to approach perception (Rieiro, Martinez-Conde, and Macknik 2013), attention (Macknik et al. 2008) and memory (Quiroga 2016) from a different perspective. However, the returned contribution to the magic community is not clear. Mostly, neuroscience has explored some techniques magicians use on stage and tried to explain them according to the scientific literature, such as saccadic movements (Cui et al. 2011; Otero-Millan et al. 2011; Kuhn and Findlay 2010; Kuhn et al. 2016; Kuhn and Teszka 2018) or psychological principles (Kuhn et al. 2014; Kuhn and Martinez 2011; Thomas, Didierjean, and Kuhn 2018). It is rare to see an approach to these techniques that is not purely phenomenological (Dehaene and Changeux 2005), and we speculate this is the reason for the small impact of this work in the magic community. Corroborating a specific technique or a maneuver with previous scientific literature can be helpful, but providing a framework to structure broad magic principles is a much more powerful tool. The literature about magic principles is vast, and it is always based on subjective experience of different magicians. In this line, a general model to explain magic principles based on neuroscience is likely to penetrate in the magic society. 
By far, misdirection is been the most studied magical principle in neuroscience (Kuhn et al. 2014; Kuhn and Martinez 2011; Cui et al. 2011; Otero-Millan et al. 2011; Kuhn and Teszka 2018; Kuhn and Findlay 2010; Wiseman and Nakano 2016). Defining misdirection with accuracy is controversial. In a previous paper, I defined it as "those actions of a magic trick that influence the attention of the spectator to conceal the method used" (Bestue, 2019). In that publication, I proposed a classification of this term based on how attention was biased generating different outputs. I differentiated between "direction" (moving attention from one initial focus to another), "division" (generating a competition between to relevant focus) and "diminishment" (reducing the detection threshold) (Bestue, 2019). Together with the definition, I provided a theoretical neuropsychological model to explain them. However, that model lacks the biological description of the processes, and needs to be implemented with well-known models with detailed circuitries.

The bump-attractor computational model (Compte et al. 2000) is been used to describe visual working memory under many circumstances (Wimmer et al. 2014; Barbosa et al., 2019; Stein et al. 2019; Almeida et al., 2015). This model can account for interference effects between competing memoranda (Almeida et al., 2015) as well as for different excitability regimes, interpretable as top-down attentional modulation. This characteristics makes the bump attractor model an excellent candidate to reproduce all different types of misdirection. In this publication, we used the bump-attractor model to reproduce all three different types of misdirection. We successfully explained in a mechanistic manner all three different types (direction, division, diminishment) providing, for the first time, neuroscientific validity for a misdirection classification.

\section{Results}

The first step before reproducing all three different types of misdirection was to simulate a control condition, where no misdirection was applied. In Bestue, 2019, I proposed 4 different cognitive modules that interplay during a magic trick (external tracking, internal tracking, evoked memories and lingering working memories). As the model we are using here is used to simulate visuospatial working memory in the current trial - although it is also been used to simulate previous trial interferences as in Barbosa \& Compte, 2018 - we are proposing a model just for the external tracking module. For the simulations, there is always going to be a "position to be misdirected away" (PMA). For simplicity, this position is arbitrary located at $180^{\circ}$, and the effectiveness of the misdirection type will be evaluated by checking if the neurons with preferred tuning for $180^{\circ}$ are active at the end of the simulation. If they are, the simulation will be label as "detected" and, if they are not, it will be labeled as "undetected". In the Bestue, 2019 I stressed out the importance of the internal dynamics, as this processes evolve with time. As a consequence, all the simulations had an arbitrary delay time of $1500 \mathrm{~ms}$, where the initial input generates a bump (ignition of activity that is maintained during the delay through reverberatory activity) that can be altered by noise or competing memories. We simulated 200 control situations, with an initial input at $180^{\circ}$. At the end of the simulation, the vast majority showed activity for the PMA (99.5\%). The remaining $0.5 \%$ are random drift of the simulation originated by noise. An example of this simulation can be found in Fig. 1A. Simulating a control condition shows that, in the absence of any type of misdirection, we can not prevent noticing whatever is at the PMA.

Once we had a control condition, we started modeling the direction type of misdirection. I previously described this type of misdirection as a focus modification of the external tracking (Bestue, 2019). An 
example of it would be when a magician emphasizes a point in the space (as a distraction) while executing a secret maneuver in another point (e.g. emphasize a coin in the right hand while the left hand does a secret maneuver). To reproduce this misdirection type in the model, we introduced an input in a "distracting location" (DL), different to the PMA (Fig. 1B). As a consequence, at the end of the simulation, the neurons coding for the PMA are less likely to be active, so misdirection works. Critically, this type of misdirection depends on the distance between the PMA and the DL. The closer is one to the other, the risk for the magician to be caught increases. We ran 200 simulations for 18 increasing distances from 10 to $180^{\circ}$ (total of 3600 simulations) and we observed that the model perfectly reproduces this feature: when increasing the separation between PMA and the $D L$, the detection dropped (Fig. 1C). The explanation is that close locations are more likely to be activated for the drift of the bump due to noise fluctuations than more distant ones. Regarding the direction type of misdirection, the model could reproduce the compromise in detection rates as well as the distance dependence.

The next type of misdirection we modeled was division. I previously described this type of misdirection as increase in load. An example of it would be when the magician executes two simultaneous action, where one of them has a secret maneuver (e.g. introducing both hands in the pocket when he wants to secretly get something from the pocket in just one of them). To reproduce this misdirection type in the model, we introduced two simultaneous inputs, one in the PMA and another in a DL, different to the PMA (Fig. 1D). In this scenario, there is a competition between both bumps to survive the delay period, and just one of them does. We ran 200 simulation and the detection rate was of $61 \%$ (there is an extra $10 \%$ to the expected $50 \%$ due to noise fluctuation). Critically, magic theory states that when regarding this type of misdirection, it is important to take into account the "priority of the movement" (Ascanio, 1964; Etcheverry, 2000). In particular, it says that when there are two competing actions for attention, the first to occur captures more attention than the second. This is also been described under the biased competition theory for visual attention (Desimone and Duncan 1995). We tested this phenomena by introducing the input of the DL before the one of the PMA (Fig. 1E). We ran an extra 200 simulations under the priority of movement condition (PM). In line with the magical theory, we observed a decrease in detection compared to the situation where competing stimuli were simultaneous (Fig. 1F). This is explained by the capacity of the circuit: it reaches a stable state with just one stimulus. When more than one are presented, there is competition until just one of them remains. When both are introduced simultaneously, there is around $50 \%$ chance for each location, but when one is introduced before the other, the first will inhibit the neurons encoding for the prefered location of the second, so it is more likely to win the competition and be maintained at the end of the simulation. Regarding the division type of misdirection, the model could reproduce the compromise in detection rates as well as the priority of movement dependence.

Finally, we modeled the diminishment type of misdirection. I previously described this type of misdirection as a change in the overall amount of attention allocated. An example of it would be when the magician executes an action in a "normal" way before executing it in a "tricky" way. (e.g. placing a coin in the left hand in a normal way before performing a "false transfer", keeping the coin in the right hand). To reproduce this misdirection type in the model, we modified the network excitability of the simulation. A key feature of the model is that it shows bistability between a resting state with spontaneous firing due to noise and a spatially structured state with sustained firing (Compte et al. 2000). The excitability of the network can be modified by changing the parameter of 
background input. This can determine in which regime of the bistability we start the simulation. If it is low, we will not find high and sustained firing that survives the delay period. If it is high, we will have spontaneous activity and sporadic false memories. Fig. $1 \mathrm{G}$ illustrates a simulation with reduced background input - low excitability -, that does not survive the delay period. Intensity of diminishment is a key feature of this type of misdirection: a strong diminishment can be found when the magician makes a joke and a small one when there is a body language relaxation. Therefore, the model should be able to reproduce this differences. We ran 200 simulations for 11 increasing background input conditions and, in line with magical theory, we observed a gradual increase in detection as background input increases (Fig. $1 \mathrm{H}$ ). Regarding the diminishment type of misdirection, the model could reproduce the compromise in detection rates as well as the dependence with the intensity of the diminishment.

\section{Discussion}

All three midirection types - direction, division and diminishment- proposed in Bestue, 2019 were explained by the bump attractor computational model. Direction was reproduced by introducing a distracting stimulus at a different location, which compromised the detection of the PMA. Distance dependence of this type of misdirection was also reproduced. Division was reproduced by introducing a competing stimulus at a different location. The competing stimulus compromised the detection of the PMA and it depended on the priority of onset, as previously proposed by literature (Ascanio, 1964). Finally, diminishment was reproduced by decreasing the excitability of the network. Intensity dependence of this type of misdirection was also reproduced. The study of misdirection lacked a mechanistic model that explains its functioning in a biological way. Here, we proposed a model that provides a mechanistic framework to understand the different types of misdirection. Thus, the misdirection classification of Bestue, 2019 is the first that has

There are some limitations we dealt with in this model: the first and most notorious one is that we just focus on the submodule of "external tracking", while there are other forms of misdirection affecting the tracking of the own generated ideas (internal tracking, Bestue, 2019). The model has to be extended to incorporate other types of misdirection as well as its interactions. Second, the model we proposed here, was created with a limited capacity of one element. This was done to reproduce the division type of misdirection in a simple way. Future work should aim for higher capacity networks that can still show this competition. Finally, the division type was reproduced by changing the background input as an approximation of an attentional suppression of the whole network. Future models could incorporate an attentional module that controls the excitability of the network externally.

The existence of a model that explains misdirection is also key for an accurate classification of it. Most misdirection classifications are based on the final output of attention or in psychological aspects (Kuhn et al. 2014; Macknik et al. 2008; Posner 1980; Theeuwes 1991; Ascanio, 1964; Bruno, 1978; Sharpe, 1988). For the first time, we focused on the dynamics that originate this different types of misdirection. With this work, we pretend to open the door to other misdirection classifications to be accompanied with a biological model that explains how its subdivisions are originated internally in the brain. In this line, the classification provided in Bestue, 2019 is the only one that has been corroborated under this approach, so it is the only one so far with supporting modeling evidence. 


\section{Bibliography}

Almeida, R., Barbosa, J., Compte, A. 2015. "Neural Circuit Basis of Visuo-Spatial Working Memory Precision: A Computational and Behavioral Study." Journal of Neurophysiology 114 (3): 1806-18. https://doi.org/10.1152/jn.00362.2015.

Ascanio, A. 1964. Consideraciones sobre la misdirection. Misdirection 1, 4-6.

Barbosa, J., Compte, A. 2018. "Build-up of Serial Dependence in Color Working Memory." BioRxiv, December. https://doi.org/10.1101/503185.

Barbosa, J., Stein, H., Martinez, R., Galan, A., Adam, K., Li, S., Valls-Solé, J., Constantinidis, C., Compte, A. 2019. "Interplay between persistent activity and activity-silent dynamics in prefrontal cortex during working memory". BioRxiv, September. doi:https://doi.org/10.1101/830471

Bestue, D. 2019. "Model of Information Process for Neuromagic." PsyArXiv, July 31. doi:10.31234/osf.io/d374z.

Bruno, J. L. 1978. Anatomy of Misdirection. Stony Brook, NY: Stoney Brook Press

Compte, .A, Brunel, N., Goldman-Rakic, P. S., Wang, XJ. 2000. "Synaptic Mechanisms and Network Dynamics Underlying Spatial Working Memory in a Cortical Network Model." Cerebral Cortex 10 (9): 910-23. https://doi.org/10.1093/cercor/10.9.910.

Cui, J., Otero-Millan, J., Macknik, S.L., King, M., Susana Martinez-Conde. 2011. "Social Misdirection Fails to Enhance a Magic Illusion." Frontiers in Human Neuroscience 5 (September): 103. https://doi.org/10.3389/fnhum.2011.00103.

Dehaene, S., Changeux, J-P. 2005. "Ongoing Spontaneous Activity Controls Access to Consciousness: A Neuronal Model for Inattentional Blindness." PLoS Biology 3 (5): e141. https://doi.org/10.1371/journal.pbio.0030141.

Desimone, R., Duncan, J. 1995. "Neural Mechanisms of Selective Visual Attention." Annual Review of Neuroscience 18: 193-222. https://doi.org/10.1146/annurev.ne.18.030195.001205.

Edin, F., Klingberg, T., Johansson, P., McNab, F., Tegnér, J., Compte, A. 2009. "Mechanism for Top-down Control of Working Memory Capacity." Proceedings of the National Academy of Sciences of the United States of America 106 (16): 6802-7. https://doi.org/10.1073/pnas.0901894106.

Etcheverry, J. 2000. La magia de Ascanio, Páginas.

Kuhn, G. 2019. Experiencing the Impossible: The Science of Magic. The MIT Press. https://doi.org/10.7551/mitpress/11227.001.0001.

Kuhn, G., Caffaratti, H., Teszka, R., Rensink, R. 2014. "A Psychologically-Based Taxonomy of Misdirection." Frontiers in Psychology 5 (December): 1392. https://doi.org/10.3389/fpsyg.2014.01392.

Kuhn, G., Findlay, J.M. 2010. "Misdirection, Attention and Awareness: Inattentional Blindness Reveals Temporal Relationship between Eye Movements and Visual Awareness." Quarterly Journal of Experimental Psychology 63 (1): 136-46. https://doi.org/10.1080/17470210902846757.

Kuhn, G., Martinez, L. M., 2011. "Misdirection - Past, Present, and the Future." Frontiers in Human Neuroscience 5: 172. https://doi.org/10.3389/fnhum.2011.00172.

Kuhn, G., Teszka, R. 2018. “Don't Get Misdirected! Differences in Overt and Covert Attentional Inhibition between Children and Adults." Quarterly Journal of Experimental Psychology 71 (3): 688-94. https://doi.org/10.1080/17470218.2016.1277770. 
Kuhn, G., Teszka, R., Tenaw, N., Kingstone, A. 2016. “Don't Be Fooled! Attentional Responses to Social Cues in a Face-to-Face and Video Magic Trick Reveals Greater Top-down Control for Overt than Covert Attention." Cognition 146 (January): 136-42. https://doi.org/10.1016/j.cognition.2015.08.005.

Macknik, S. L., King, M., Randi, J., Robbins, A., Teller, Thompson, J., Martinez-Conde, S. 2008. "Attention and Awareness in Stage Magic: Turning Tricks into Research." Nature Reviews. Neuroscience 9 (11): 871-79. https://doi.org/10.1038/nrn2473.

Martinez-Conde, S., Macknik, S. L. 2008. "Magic and the Brain." Scientific American 299 (6): 72-79. https://doi.org/10.1038/scientificamerican1208-72.

Otero-Millan, J., Macknik, S. L., Robbins, A., Martinez-Conde, S. 2011. "Stronger Misdirection in Curved than in Straight Motion." Frontiers in Human Neuroscience 5 (November): 133. https://doi.org/10.3389/fnhum.2011.00133.

Posner, M I. 1980. "Orienting of Attention." The Quarterly Journal of Experimental Psychology 32 (1): 3-25. https://doi.org/10.1080/00335558008248231.

Quiroga, R. Q. 2016. “Magic and Cognitive Neuroscience." Current Biology 26 (10): R390-4. https://doi.org/10.1016/j.cub.2016.03.061.

Rieiro, H., Martinez-Conde, S., Macknik, S. L. 2013. “Perceptual Elements in Penn \& Teller's 'Cups and Balls' Magic Trick." PeerJ 1 (February): e19. https://doi.org/10.7717/peerj.19.

Sharpe, S. H. 1988. Conjurers' Psychological Secrets. Calgary, AB: Hades Publications.

Stein, H. Barbosa, J. Rosa-Justicia, Prades, L. Morató, A. Galan, A. Ariño, H. Martinez-Hernandez, E. Castro-Fornieles, J. Dalmau, J. Compte, A. 2019. "Disrupted serial dependence suggests deficits in synaptic potentiation in anti-NMDAR encephalitis and schizophrenia." PsyArXiv, doi: https://doi.org/10.1101/830471

Theeuwes, J. 1991. "Exogenous and Endogenous Control of Attention: The Effect of Visual Onsets and Offsets." Perception \& Psychophysics 49 (1): 83-90. https://doi.org/10.3758/BF03211619.

Cyril, T., Didierjean, A., Kuhn, G. 2018. "It Is Magic! How Impossible Solutions Prevent the Discovery of Obvious Ones?" Quarterly Journal of Experimental Psychology 71 (12): 174702181774343. https://doi.org/10.1177/1747021817743439.

Wimmer, K., Nykamp, D. Q., Constantinidis, C., Compte, A. 2014. "Bump Attractor Dynamics in Prefrontal Cortex Explains Behavioral Precision in Spatial Working Memory." Nature Neuroscience 17 (3): 431-39. https://doi.org/10.1038/nn.3645.

Wiseman, R., Nakano, T. 2016. "Blink and You'll Miss It: The Role of Blinking in the Perception of Magic Tricks." PeerJ 4 (April): e1873. https://doi.org/10.7717/peerj.1873.

\section{Acknowledgements}

I would like to thank PhD. Luis Martínez Otero, PhD. Albert Compte and PhD. Jordi Camí, who supported me with insight and expertise during the research. 


\section{Figures}

A

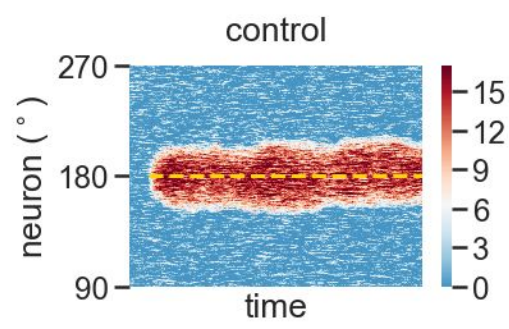

B

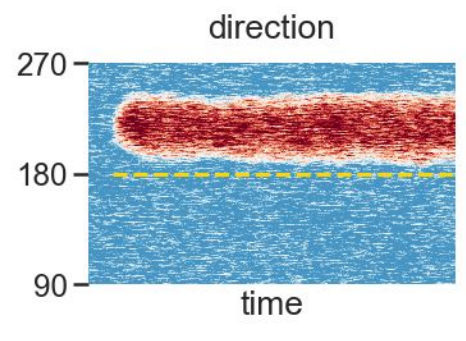

C

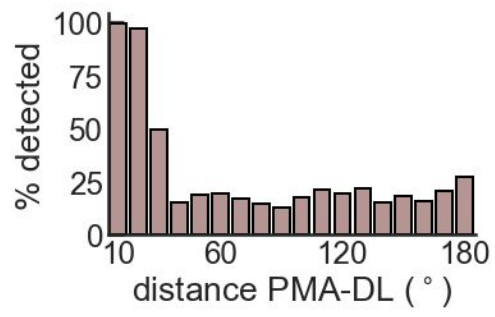

D

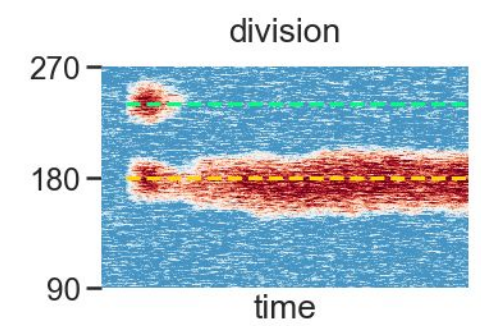

E

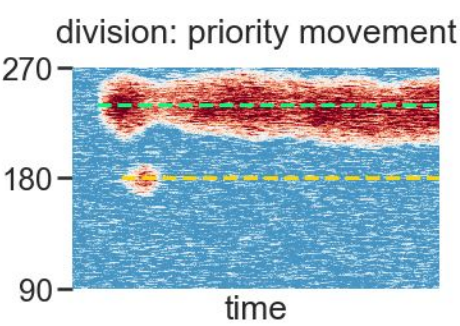

F

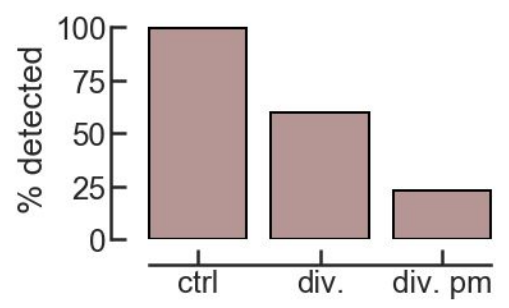

G

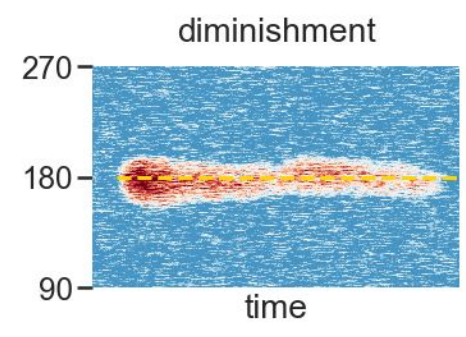

H

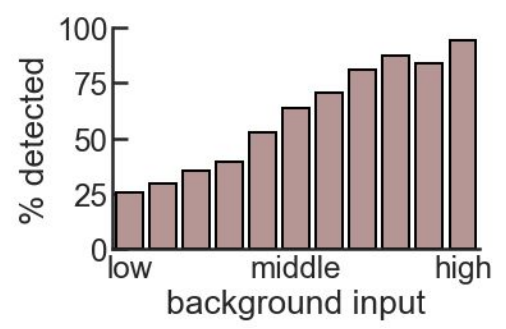

Figure $1 \mathrm{~A}$ ) Example of control simulation. The yellow line indicates the location to be misdirected away. As no misdirection technique is applied, the final decoding location of the bump contains the location. When that is the case, we will consider that the spectator was not misdirected. B) Example of misdirection by direction. C) Misdirection by detection depends on the distance between the location to be "position to be misdirected away" (PMA) and the "distracting position" (DL) of the stimulus that captures attention. D) Example of misdirection by division. The green line represents the competing location E) Example of misdirection by division applying the concept of "priority of action". F) Comparison in detection rate for misdirection by division. When applying the "priority of action", misdirection is more effective. G) Example of misdirection by diminishment. H) Misdirection by diminishment depends on the background input. 


\section{Model}

A rate version of the bump attractor (Compte et al. 2000; Edin et al. 2009) was used to reproduce different misdirection types. The bump attractor model is a Wilson-Cowan model, formed by a population of excitatory neurons and a population of inhibitory neurons, interconnected and with selectivity for specific locations in the visual space. The neurons of the model are tuned, so neurons with similar selectivity are strongly connected than the ones coding for distant positions. As a consequence of this connectivity, a ring structure emerges. In the absence of the stimulus, information is kept through reverberatory activity, maintaining the information in the form of a bump.

The model defines two different currents: excitatory and inhibitory. Each one is modeled as the sum of the external input, the background input (which defines the excitability of the network) and the weighted sum of the connectivity with rate. Each weighted sum is modulated by a parameter of conductance depending on the direction of the connectivity (excitatory to excitatory, excitatory to inhibitory, inhibitory to excitatory or inhibitory to inhibitory) .

The model describes a decrease of rate in the absence of external current modulated by temporal variables. Uncorrelated white gaussian noise is incorporated to both excitatory and inhibitory populations, allowing the bumps to drift with time. The connectivity between and within populations follows a Von mises distribution.

The final readout position is obtained by computing the population vector in the case of a single bump or by fitting a bi-von misses in the simulations with more than one bump. The effectiveness of the misdirection method was computed by getting the error from the final decoded position to the PMA specified at the beginning of each simulation. 